\title{
REVIEW \\ Ecophysiological Traits and Genetic Analysis of Yield and Ripening in High-Yielding Semi-Dwarf Indica Rice Varieties
}

\author{
Kenji NAGATA* \\ Department of Paddy Farming, National Agricultural Research Center for Tohoku Region \\ (Daisen, Akita 014-0102, Japan)
}

\begin{abstract}
Experiments were conducted to examine the ecophysiological traits of yield and ripening and the genetic backgrounds of these traits in high-yielding semi-dwarf indica rice. Field experiments were performed over four years to examine the yield and growth characteristics of these traits. A large sink size with large numbers of spikelets per panicle and a high ripening ability in the background of a large sink size were found to be two of the major ecophysiological traits that enhance the yield of semidwarf indica. High dry matter productivity after heading was suggested to be linked to a high ripening ability. To determine the genetic backgrounds that control these traits, quantitative trait locus (QTL) analysis was conducted using two different semi-dwarf indica/japonica inbred lines: recombinant inbred lines of Milyang23 (semi-dwarf indica)/Akihikari (japonica), and back-crossed inbred lines of Sasanishiki (japonica)/Habataki (semi-dwarf indica)//Sasanishiki///Sasanishiki. QTLs for larger sink size, higher ripening ability, higher dry matter production, and larger nonstructural carbohydrate (NSC) reserves in leaf sheaths and culms at heading within semi-dwarf indica alleles were detected. The effects of these QTLs probably combine to express the large sink size and high ripening ability traits, resulting in the high yields of the semi-dwarf indica rice varieties.
\end{abstract}

Discipline: Crop production / Plant breeding

Additional key words: dry matter production, nonstructural carbohydrate (NSC), quantitative trait locus (QTL), sink size

\section{Introduction}

Rice (Oryza sativa L.) is one of the world's most important crops, especially in Asia. Nearly $90 \%$ of global rice production comes from Asia, since $32 \%$ of calorie intake of Asian people depends on rice consumption ${ }^{2}$. Yields of paddy rice in Asia have doubled in the past 40 years, from 1.9 (1961-1963) to $4.0 \mathrm{t} \mathrm{ha}^{-1}(2001-2003)^{2}$. However, the increase in yield from 1990 to 2003 was only $0.7 \%$ per year, much lower than the increase in the human population $(1.6 \%)^{2}$. Therefore, it is still important to enhance rice yields and production.

Semi-dwarf indica varieties have played important roles in increasing the yield potential of rice plants. Following the release of IR8 from IRRI, numerous pedigrees have been developed and cultivated in many monsoon Asian countries, contributing to the food security of these countries. Semi-dwarf indica varieties, which have also been developed in Japan ${ }^{7}$, exhibit higher yields than japonica varieties ${ }^{6}$. Although much research has been conducted on the ecophysiological factors associated with the high yield of the semi-dwarf indica varieties ${ }^{6,8,11}$, the genetic basis of the high yields has not yet been clarified. Such genetic information would be very useful for the efficient production of high-yielding varieties in breeding programs, as well as for analyzing the exact nature of the processes that determine yield in the rice plant.

This study examined ecophysiological traits related to yield in high-yielding semi-dwarf indica rice varieties in Japan. Quantitative trait locus (QTL) analysis was conducted using semi-dwarf indica/japonica cross populations to clarify the genomic regions linked to these traits. 


\section{Ecophysiological traits related to yield in high- yielding semi-dwarf indica rice}

Field experiments were conducted to elucidate the traits that enhance yield in high-yielding semi-dwarf indica rice ${ }^{13}$. Japonica and semi-dwarf indica varieties were cultivated from 1992 to 1995 at the Shikoku National Agricultural Experiment Station (Kagawa, Japan). Seedlings were transplanted in late May, and the plants were cultivated under conditions of high nitrogen application, with a total of $12 \mathrm{~g} \mathrm{~m}^{-2}$ applied from 1992 to 1994, and $18 \mathrm{~g} \mathrm{~m}^{-2}$ in 1995.

The average yield of rough brown rice in the semidwarf indica crop through the 4-year study was $836 \mathrm{~g} \mathrm{~m}^{-2}$, $30 \%$ higher than that of the japonica crop (Table 1). The highest yield, $1,035 \mathrm{~g} \mathrm{~m}^{-2}$, was produced by the semidwarf indica variety Saikai198 in 1994. In spite of bearing fewer panicles per plant, the semi-dwarf indica produces a much larger number of spikelets per panicle, leading to more spikelets and a larger sink size per land area. Although an increase in the sink size often results in reduced ripening percentages in rice ${ }^{10,19}$, the ripening percentages of the semi-dwarf indica did not show significant changes. This result is also confirmed by Fig. 1, which shows the relationship between sink size and ripening. In the japonica varieties, there was a clear negative correlation between the two traits throughout the years tested. In contrast, the semi-dwarf indica varieties did not show such a reduction in the ripening percentages, in spite of having larger sink sizes than the japonica varieties.

Examination of growth traits showed that the semidwarf indica had a shorter culm length, a longer panicle length, a higher dry weight at maturity, and longer growth periods for both the days from transplanting to heading and the grain-filling duration (Table 2). The longer growth period of the semi-dwarf indica could contribute to the greater dry weight. In Fig. 1, data plots of the growth of the semi-dwarf indica variety Kanto147 and those of the semi-dwarf indica obtained in the 1993 experiment did not show the high ripening abilities. These results are likely to be due to the highly unfavorable conditions in 1993, including low temperatures and low solar irradiations through the growing period, and the low dry matter production of Kanto147 during the ripen-

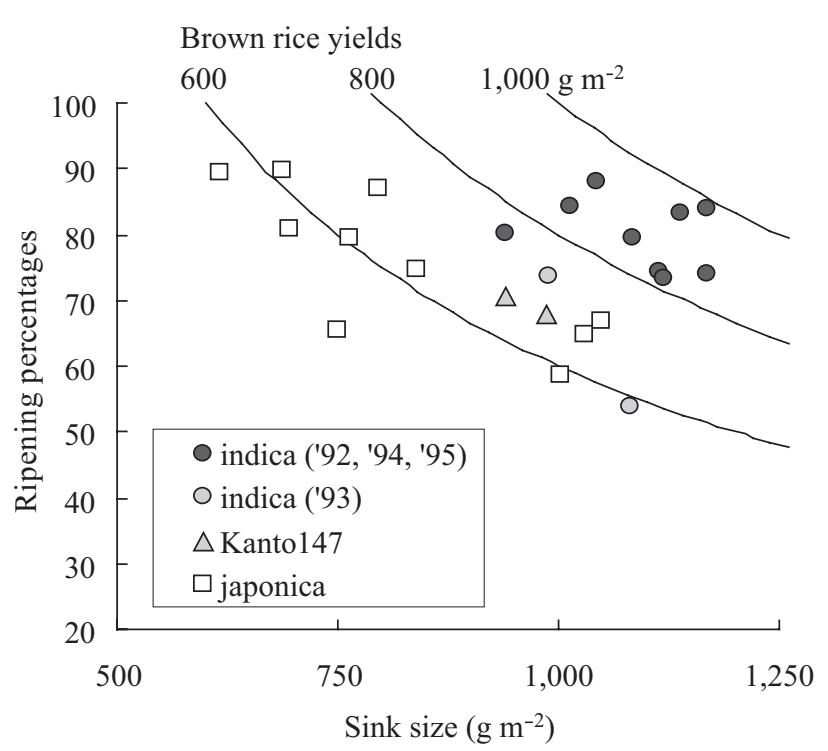

Fig. 1. Relationship between sink size and ripening percentages

Sink size was calculated by multiplying the number of spikelets $\mathrm{m}^{-2}$ by 1,000 grains weight.

Table 1. Yield and its components of japonica and semi-dwarf indica rice varieties tested during 1992-1995

\begin{tabular}{|c|c|c|c|c|c|c|c|c|c|}
\hline Type & & $\begin{array}{l}\text { Rough brown rice } \\
\text { yield }{ }^{\text {a) }}\left(\mathrm{gm}^{-2}\right)\end{array}$ & $\begin{array}{l}\text { Brown rice } \\
\text { yield }^{\mathrm{a}}\left(\mathrm{gm}^{-2}\right)\end{array}$ & $\begin{array}{l}\text { Panicle no. } \\
\qquad\left(\mathrm{m}^{-2}\right)\end{array}$ & $\begin{array}{l}\text { Spikelets per } \\
\text { panicle }\end{array}$ & $\begin{array}{l}\text { Spikelet no. } \\
\left(\times 10^{3} \mathrm{~m}^{-2}\right)\end{array}$ & $\begin{array}{l}\text { 1,000 grains } \\
\text { weight }^{\mathrm{a})}(\mathrm{g})\end{array}$ & $\begin{array}{l}\left.\text { Sink size }{ }^{\mathrm{b}}\right) \\
\left(\mathrm{g} \mathrm{m}^{-2}\right)\end{array}$ & $\begin{array}{l}\text { Ripening }^{c)} \\
(\%)\end{array}$ \\
\hline Japonica & $\max$ & 815 & 700 & 454 & 138 & 52.0 & $39.1^{\mathrm{e})}$ & 1,052 & 89.8 \\
\hline \multirow[t]{2}{*}{$(\mathrm{n}=10)$} & $\min$ & 549 & 531 & 285 & 72 & 21.5 & 19.8 & 617 & 58.8 \\
\hline & ave & 643 & 609 & 370 & 92 & 34.0 & 25.0 & 829 & 75.7 \\
\hline \multirow{4}{*}{$\begin{array}{l}\text { Semi-dwarf } \\
\text { indica } \\
(\mathrm{n}=13)\end{array}$} & $\max$ & 1,035 & 979 & 380 & 183 & 55.9 & 24.4 & 1,167 & 87.9 \\
\hline & $\min$ & 621 & 579 & 294 & 124 & 40.4 & 19.1 & 939 & 53.7 \\
\hline & ave & 836 & 799 & 330 & 149 & 48.9 & 21.8 & 1,061 & 75.9 \\
\hline & $t$-test ${ }^{\mathrm{d})}$ & $* * *$ & $* * *$ & * & $* * *$ & $* * *$ & $\mathrm{~ns}$ & $* *$ & ns \\
\hline
\end{tabular}

Data of the early season transplanting cultivation obtained in Nagata et al. ${ }^{13}$ was summarized. "Japonica" includes the varieties Fukuhibiki (Ouu331), Ouu342, Oochikara, Kanto158, Kanto176, Hokuriku153, and Nipponbare. "Semi-dwarf indica" includes the varieties Kanto147, Takanari, Kou266, Saikai198, and Saikai203.

a): Water content was adjusted to $15 \%$ of dry weight.

b): Calculated by multiplying the number of spikelets $\mathrm{m}^{-2}$ by 1,000 grains weight.

c): Selected by water soaking.

d): $* * *, * *$ and $*$ indicate that the averages are different significantly at $0.1,1$ and $5 \%$ levels, respectively; ns, not significant.

e): Including big grain varieties such as Oochikara and Hokuriku153. 
Table. 2 Growth characteristics of japonica and semi-dwarf indica rice varieties tested during 1992-1995

\begin{tabular}{llccccccc}
\hline \hline Type & & $\begin{array}{c}\text { Days to } \\
\left.\text { heading }^{\mathrm{a}}\right)\end{array}$ & $\begin{array}{c}\text { Days to } \\
\text { maturity }\end{array}$ & $\begin{array}{c}\text { Culm length } \\
(\mathrm{cm})\end{array}$ & $\begin{array}{c}\text { Panicle } \\
\text { length }(\mathrm{cm})\end{array}$ & $\begin{array}{c}\text { Dry weight at } \\
\text { maturity }^{\mathrm{b}}\left(\mathrm{g} \mathrm{m}^{-2}\right)\end{array}$ & $\begin{array}{c}\text { Lodging } \\
(0-4)^{\mathrm{c})}\end{array}$ & $\begin{array}{c}\text { Harvest } \\
\text { index }^{\mathrm{d})}\end{array}$ \\
\hline Japonica & $\max$ & 81 & 127 & 88.9 & 25.5 & 1,992 & 2.4 & 0.51 \\
$(\mathrm{n}=10)$ & $\min$ & 62 & 97 & 66.2 & 18.6 & 1,221 & 0.0 & 0.33 \\
& ave & 73 & 115 & 79.8 & 21.7 & 1,653 & 0.8 & 0.39 \\
Semi-dwarf & $\max$ & 90 & 144 & 75.0 & 27.5 & 2,403 & 1.0 & 0.45 \\
indica & $\min$ & 73 & 114 & 65.9 & 22.1 & 1,535 & 0.0 & 0.30 \\
$(\mathrm{n}=13)$ & ave & 82 & 131 & 70.7 & 25.3 & 2,083 & 0.1 & 0.40 \\
& $t$-test $\left.{ }^{\mathrm{e}}\right)$ & $* *$ & $* * *$ & $* *$ & $* * *$ & $* * *$ & $\mathrm{~ns}$ & $\mathrm{~ns}$ \\
\hline
\end{tabular}

Data of the early season transplanting cultivation obtained in Nagata et al. ${ }^{13}$ was summarized. Details are described in Table 1.

a): Days after transplanting.

b): Air-drying.

c): 0 ; no lodging to 4 ; complete lodging.

d): Ratios of the rough brown rice yield to the dry weight at maturity.

e): ${ }^{* *}$ and $* *$ indicate that the averages are different significantly at 0.1 and $1 \%$ levels, respectively; ns, not significant.

ing period due to rapid leaf senescence after heading ${ }^{12}$. These facts indicate that the source supply during the ripening period is an important factor in the high ripening ability of the semi-dwarf indica. Therefore, higher dry matter production after heading could be responsible for high yields.

These experiments indicate that the high-yielding semi-dwarf indica rice achieves high yields in Japan due to the following traits:

- Large sink size with a large number of spikelets per panicle

- High ripening ability under large-sink-size conditions The high dry matter productivity after heading, with or without a long growth duration, was suggested to be linked to the high ripening ability when the source supply is sufficient during the ripening period.

\section{QTL analysis for sink size, ripening and source ability of semi-dwarf indica rice}

To determine the genomic regions responsible for the traits of enhanced yield and ripening ability of semidwarf indica rice, QTL analysis was conducted with two semi-dwarf indica/japonica inbred lines ${ }^{14}$. Recombinant inbred lines (RILs) of Milyang23 (semi-dwarf indica)/ Akihikari (japonica) ${ }^{4}$ as well as back-crossed inbred lines (BILs) of Sasanishiki (japonica)/Habataki (semi-dwarf indica)//Sasanishiki///Sasanishiki (Sasanishiki $\times$ Habataki) ${ }^{5}$ were cultivated in the Hokuriku National Agricultural Experiment Station (Niigata, Japan) for two years, in 1997 and 1998 for the RILs, and in 1998 and 1999 for the BILs, respectively. The results of interval mapping for the QTL analysis of the RILs and the BILs for the sink size and ripening ability are shown in Figs. 2 and 3, respectively.

The sink size is almost determined by the number of spikelets per plant, which is the product of the number of spikelets per panicle and the number of panicles per plant. In semi-dwarf indica, a higher number of spikelets per panicle had a strong positive impact on the sink size, as shown above (Table 1). Two and four QTLs for the number of spikelets per panicle, indica (Milyang23 or Habataki) alleles which increased the trait, were detected in the RILs and the BILs, respectively. Among them, an important region was found on the short arm of chromosome 1 that increased the number of spikelets per panicle and the sink size (Figs. $2 \& 3$ ). The effect of this QTL on the number of spikelets per panicle was very strong and was also consistent in the two different populations of the RILs and the BILs; the percentage of variance explained was $29.5-37.2 \%$ for the RILs and $31.3-34.3 \%$ for the BILs. Therefore, this locus appears to be ubiquitous in high-yielding semi-dwarf indica varieties and has been used to increase the number of spikelets in recently bred high-yielding indica-japonica crossed varieties in $\operatorname{Japan}^{20}$.

Despite the strong positive effect on the number of spikelets per panicle, this QTL on chromosome 1 had pleiotropic effects, reducing the number of panicles per plant and the ripening percentages. In contrast, a QTL on chromosome 6 detected in the BILs increased the number of spikelets per panicle with little or no influence on other traits, including the number of panicles per plant and the ripening percentages (Fig. 3). The differing effects of the QTLs on chromosomes 1 and 6 on ripening abilities may be due to morphological differences in the panicles. The higher number of spikelets per panicle in plants containing the indica allele of the chromosome-1 QTL is related to increased numbers of secondary rachis branches (Figs. 

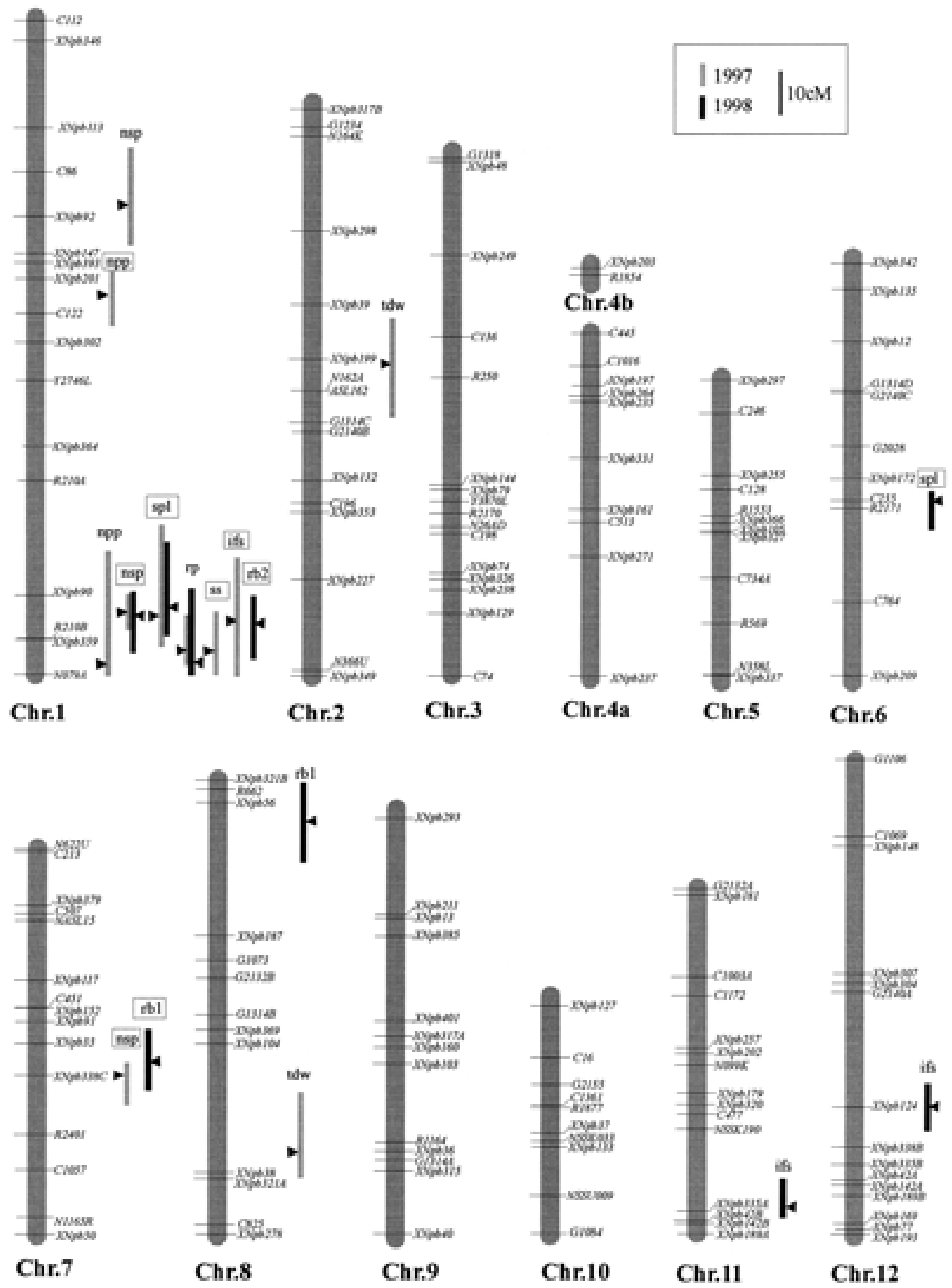

Fig. 2. RFLP linkage map showing the locations of quantitative trait loci (QTLs) for the traits related to sink size and ripening for the RILs of rice derived from the cross Milyang23/Akihikari

Markers are shown on the right side of the chromosomes. A LOD score of 3.0 was used to detect putative QTLs. Arrowheads indicate the peak positions of the LOD, and gray and black bars indicate the regions with one LOD support interval in the 1997 and 1998 experiments, respectively. Trait designations are abbreviated as follows: npp, number of panicles per plant; nsp, number of spikelets per panicle; spl, number of spikelets per plant; rp, ripening percentage; tdw, total dry weight at maturity; ss, percentage of sterile spikelets; ifs, percentage of insufficiently filled spikelets; rb1, number of primary rachis branches; and rb2, number of secondary rachis branches. Abbreviations enclosed in boxes indicate the traits that increased in the Milyang23 alleles and the others in the Akihikari alleles. The chromosomes are arranged so that the short arm is at the bottom. Details for the locations and the effects of each QTL are described in Nagata et al. ${ }^{14}$. 


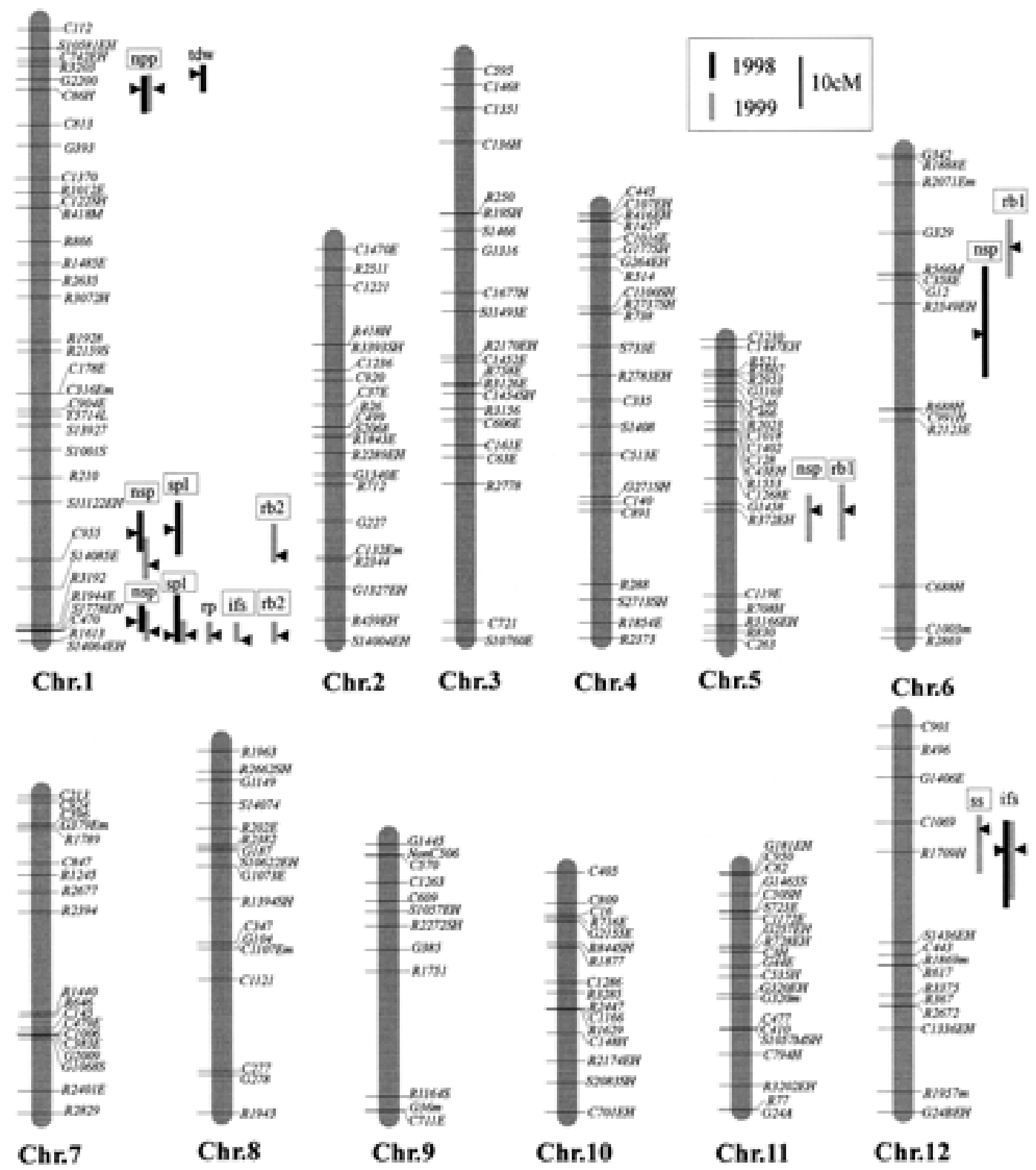

Fig. 3. RFLP linkage map showing the locations of QTLs for the traits related to sink size and ripening in the BILs developed from the cross Sasanishiki/Habataki//Sasanishiki///Sasanishiki

The black and gray bars indicate the regions with one LOD support interval obtained in the 1998 and 1999 experiments, respectively. Abbreviations enclosed in boxes indicate the traits that increased in the Habataki alleles and the others in the Sasanishiki alleles. Other explanations are the same as those in Fig. 2.

2 \& 3). In contrast, the QTL on chromosome 6 mainly acts to increase the number of primary rachis branches (Fig. 3). This observation might explain the different effects of the two QTLs on ripening, since the spikelets on secondary rachis branches often show poorer ripening ability than those on primary rachis branches ${ }^{1,19}$. Indica alleles of QTLs that were found on chromosome 7 in the RILs and on chromosome 5 in the BILs increased both the number of spikelets per panicle and the number of primary rachis branches (Figs. $2 \& 3$ ). However, these
QTLs had little effect on the sink size because their effect on the number of spikelets per panicle was relatively small, and they also reduced the number of panicles per plant.

In the Milyang23/Akihikari RILs, QTLs for the decreased percentages of insufficiently filled spikelets with indica alleles that were independent of the sink size were detected on chromosomes 11 and 12 (Fig. 2). Although they were observed less consistently and require further investigation, these loci could be candi- 
dates for traits that enhance the ripening ability of semidwarf indica rice varieties.

QTLs for dry matter accumulation were detected on chromosomes 2 and 8 in the RILs and chromosome 1 in the BILs (Figs. $2 \& 3$ ). However, indica alleles of each of these loci showed negative effects on dry weight at maturity. To determine the chromosome regions that increase the dry matter productivity in indica genotypes, the accu- mulation of dry matter before and after heading was evaluated in 1998 and 2000 using the Sasanishiki $\times$ Habataki BILs. From this analysis, a QTL for increased total dry weight both at heading and at maturity with an indica allele was detected on chromosome 12 in 2000 (Fig. 4). The effect of this QTL for dry matter accumulation might be due to the pleiotropic effect on the number of days to heading (Fig. 4), suggesting that a longer growth duration

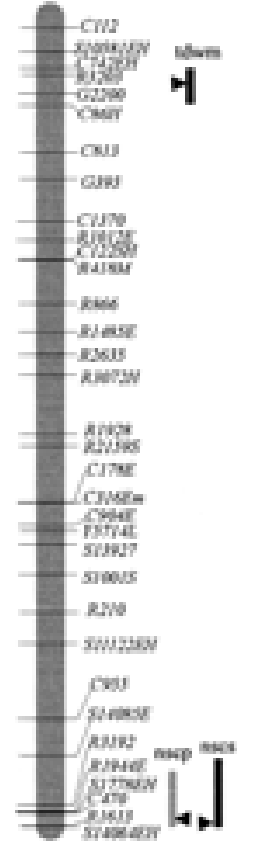

Chr.1

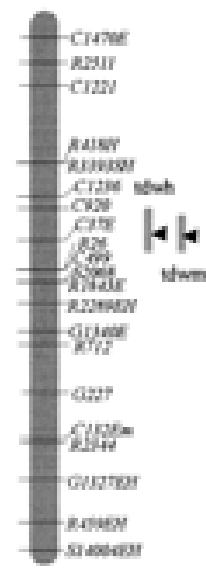

Chr.2

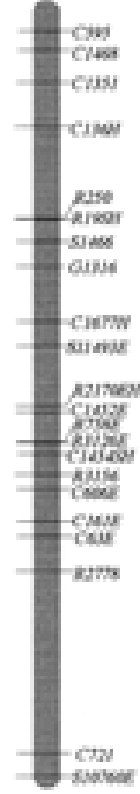

Chr.3

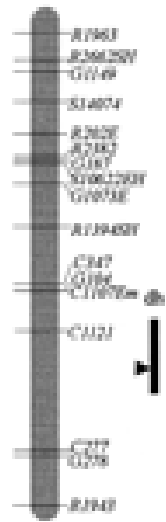

Chr.8

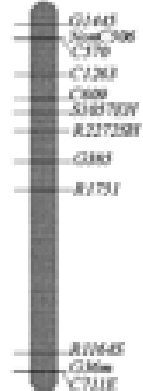

Chr.9

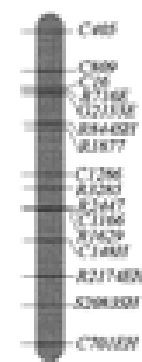

Chr.10
Chr.11
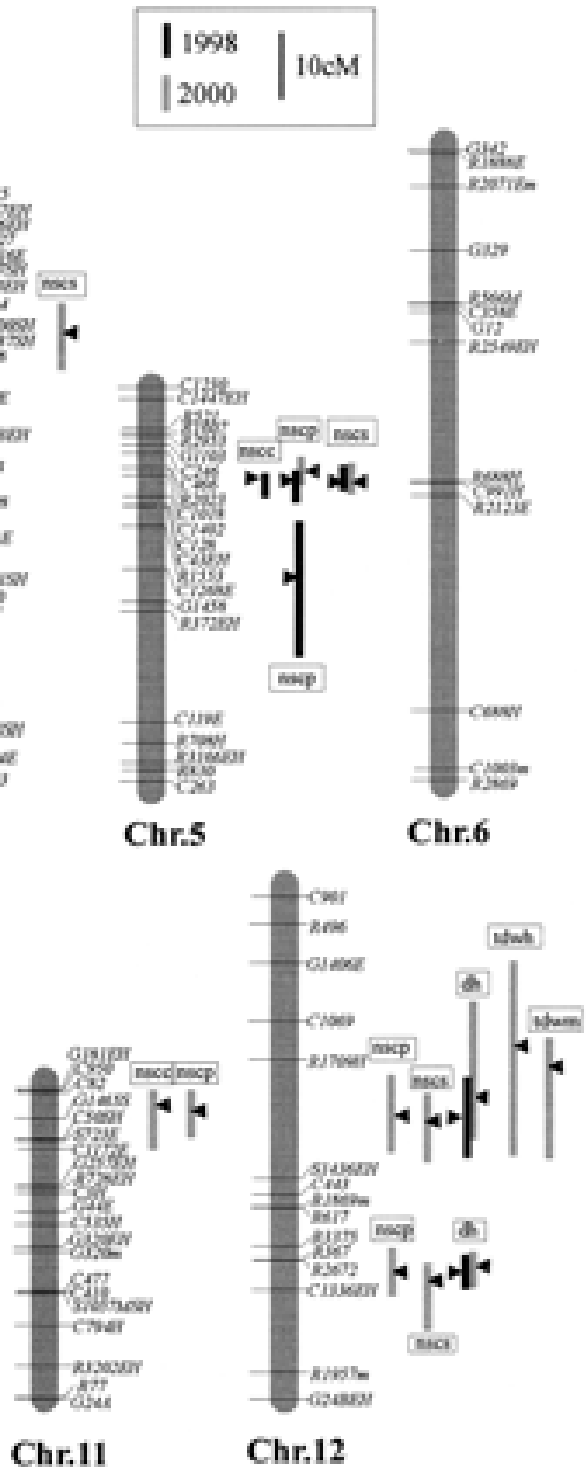

Fig. 4. RFLP linkage map showing the locations of QTLs for the traits related to dry matter production, NSC accumulation and the number of days to heading for the BILs of rice derived from the cross Sasanishiki/Habataki//Sasanishiki/// Sasanishiki

The black and gray bars indicate the regions with one LOD support interval in the 1998 and 2000 experiments, respectively. Trait designations are abbreviated as follows: tdwh, total dry weight at heading; tdwm, total dry weight at maturity; nscc, NSC content; nscp, total amount of NSCs per plant; nscs, amount of NSCs per spikelet; and dh, number of days to heading. Abbreviations enclosed in boxes indicate the traits that increased in the Habataki alleles and the others in the Sasanishiki alleles. Details for the locations and the effects of each QTL are described in Nagata et al. ${ }^{15}$. Other explanations are the same as those in Fig. 2. 
causes a greater dry matter accumulation. In addition to this QTL, there were several regions that resulted in increased dry weight accumulation when the regions were derived from indica, although the effects were relatively small (Fig. 5). Among them, a region on chromosome 5 was detected consistently; this region might be responsible for the high dry matter accumulation of the semi-dwarf indica rice.

Nonstructural carbohydrates (NSCs) accumulated in leaf sheaths and culms at heading are translocated to panicles and used in grain development after heading, which have been reported to play important roles in ripening abilities as source supply during ripening period in rice $^{17,18}$. Analysis using the Sasanishiki $\times$ Habataki BILs in 1998 and $2000^{15}$ revealed several significant regions that affect NSC accumulation at heading, located on chromosomes 1, 4, 5, 7, 11, and 12 (Fig. 4). The QTLs that affect NSC accumulation on chromosomes 7 and 12 coincide with QTLs for the number of days to heading
(Fig. 4), strongly suggesting that these QTLs have pleiotropic effects. The indica alleles of these loci increased NSC accumulation with delayed heading, suggesting that a longer period of vegetative growth before heading might cause higher NSC accumulation. In contrast, the QTLs on chromosomes 1, 4, 5, and 11 had no effect on the number of days to heading. Of these, the japonica allele of the QTL on chromosome 1 increased NSC accumulation (Fig. 4); this QTL might be identical to the QTL for which the indica allele drastically increased the number of spikelets per plant and reduced the ripening percentage (Figs. 2, $3 \& 6$, A1 \& B1). This suggests that the increase in the number of spikelets per panicle in the indica allele due to this locus results in decreased NSC accumulation, probably because some of the NSCs are used in the production of larger panicles during the panicle-development period before heading. In addition, the low NSC accumulation might be one of the reasons for the poor grain filling in plants containing the indica allele

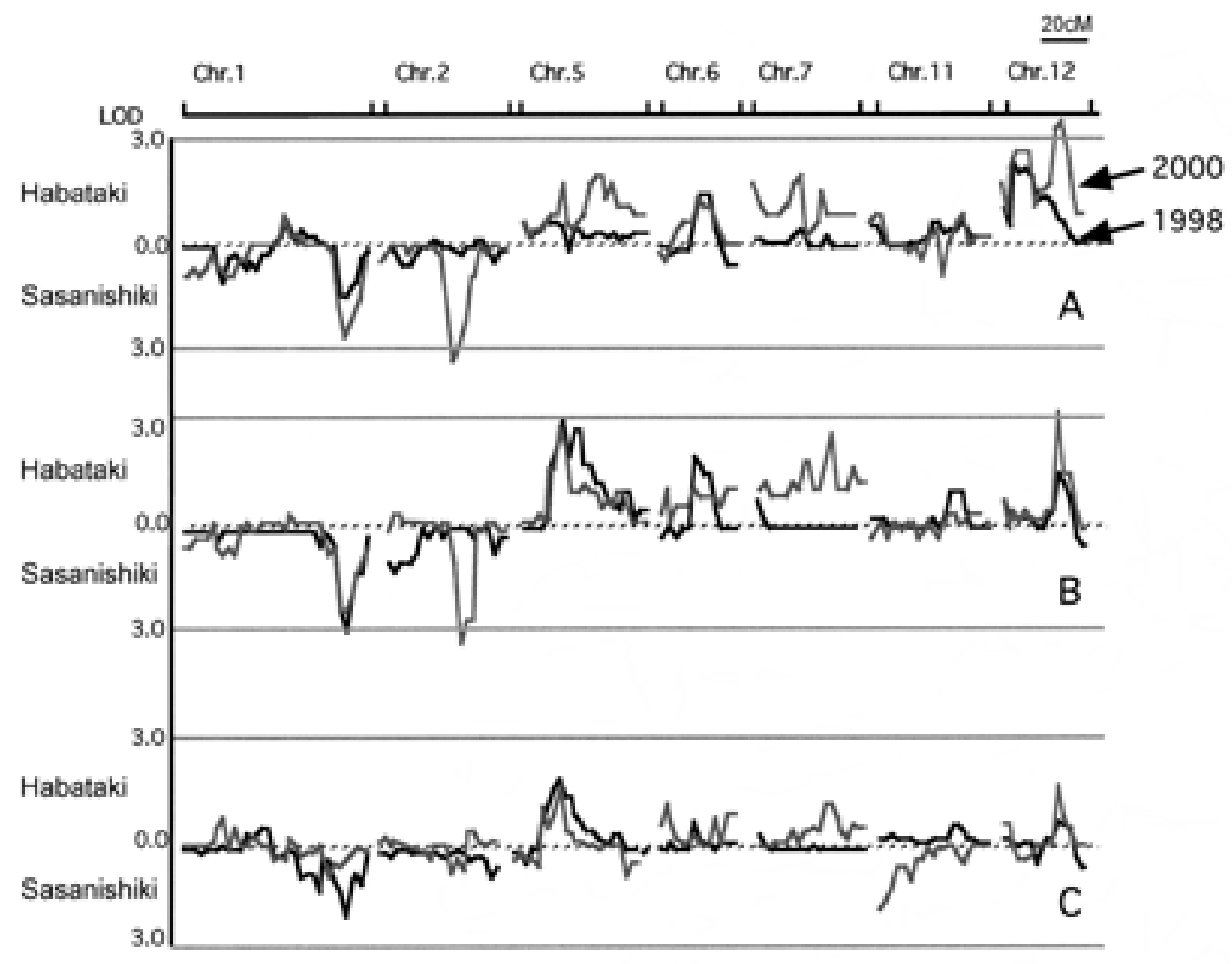

Fig. 5. Interval mapping of QTL analysis on chromosomes $1,2,5,6,7,11$, and 12 for the traits related to dry matter production for the BILs from the cross Sasanishiki/Habataki//Sasanishiki///Sasanishiki

A: Total dry weight at heading, B: Total dry weight at maturity, C: Dry weight increase from heading to maturity. Black and gray lines indicate the LOD scores for the 1998 and 2000 experiments, respectively. LOD scores of loci showing positive and negative additive effects with the Habataki allele are indicated above and below, respectively. Chromosomes are arranged so that the short arm is on the left side. Thin lines indicate significant levels for LOD score $=3.0$. 
of this locus (Figs. $3 \& 6, \mathrm{C} 1$ ). In contrast, the QTLs for NSC accumulation on chromosomes 5 and 11 had little effect on the sink size (Fig. 6, A5 \& A11), but did have some effect on the number of insufficiently filled spikelets (Fig. 6, B5 \& B11). The higher amount of NSCs accumulated before heading, resulting from the indica alleles of these loci, reduced the percentage of insufficiently filled spikelets. Therefore, these QTLs for NSC accumulation might provide an important contribution to the high ripening ability of semi-dwarf indica rice varieties. The QTL detected on chromosome 4 might also be useful, but its effects were not consistent and need to be confirmed.

$S d 1$, a major gene for semi-dwarfism in rice, has been mapped ${ }^{3,9}$ to the long arm of chromosome 1 near the marker $C 86$ and cloned ${ }^{16}$. In this study, higher numbers of panicles per plant were observed in plants containing the indica allele of this region (Fig. 3). However, lower numbers of spikelets per panicle and lower dry matter accumulation, which might have detrimental effects on yield, were also observed in plants containing this region (Figs. $2,3 \& 4$ ). Although a more precise analysis of the linkage between these traits and semi-dwarfism should be performed, the high yield of the semi-dwarf indica rice varieties used in this analysis appears to be due to genomic regions other than the $s d l$ locus.

\section{Conclusions and perspectives}

The results obtained in this study are summarized in Fig. 7. The QTL analysis indicates that the large sink size and the high ripening ability in the high-yielding semi-dwarf indica rice varieties result from the controls of many genetic loci. In the future, it will be important to investigate the precise physiological functions of each QTL. Research in this area is underway using chromo-

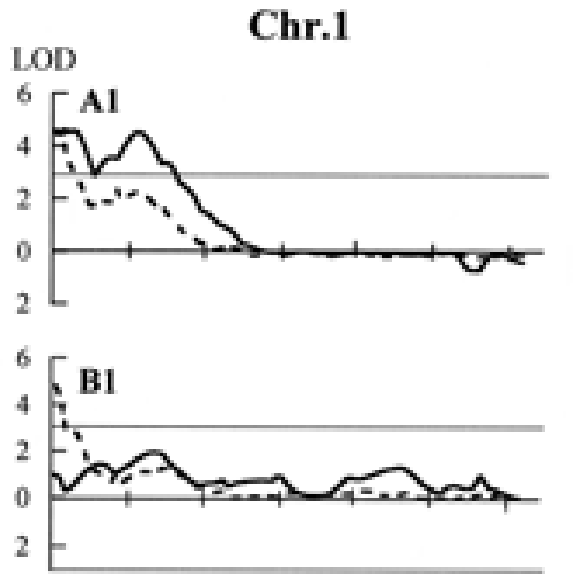

Chr.5
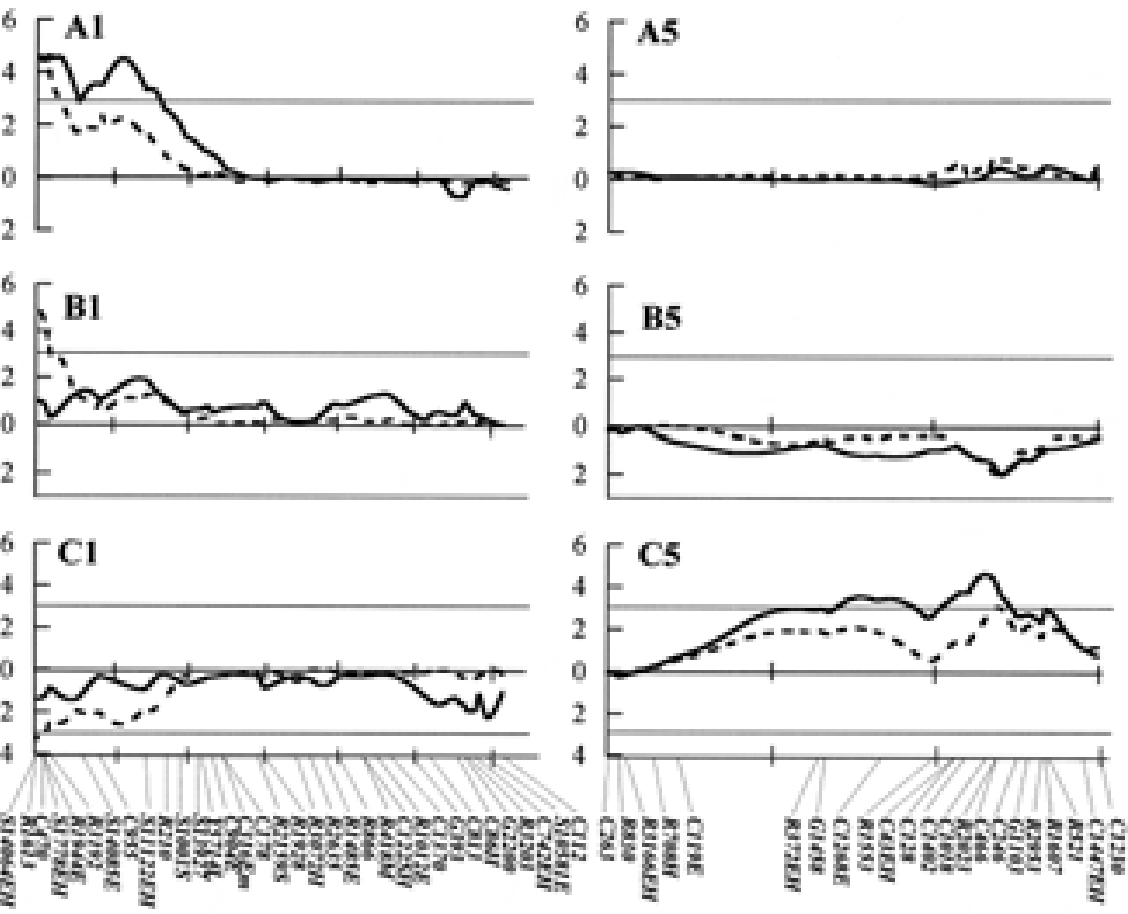

Chr.11
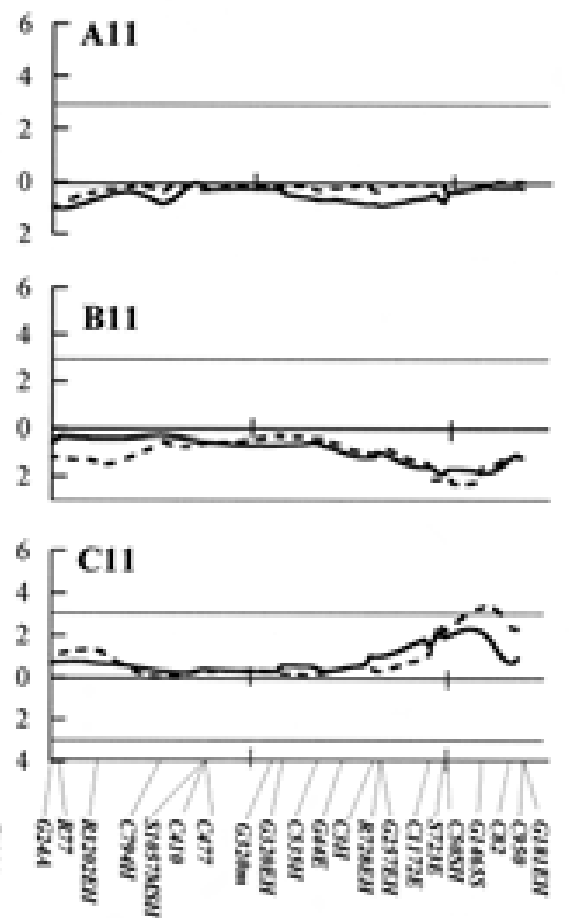

Fig. 6. Interval mapping of QTL analysis on chromosomes 1, 5 and 11 for the traits related to sink size, incompletely filled spikelets and total amount of NSCs for the BILs from the cross Sasanishiki/Habataki//Sasanishiki///Sasanishiki

A: Number of spikelets per plant, B: Percentage of incompletely filled spikelets, C: Total amount of NSCs per plant. The suffixes 1, 5 and 11 denote chromosomes 1, 5 and 11, respectively. A and B were plotted using data obtained in Fig. 3, whereas $\mathrm{C}$ was plotted using data obtained in Fig. 4. Solid and broken lines in A and B indicate the LOD scores for the 1998 and 1999 experiments, whereas those in C indicate the LOD scores for the 1998 and 2000 experiments, respectively. LOD scores of loci showing positive and negative additive effects with the Habataki allele are indicated above and below, respectively. Chromosomes are arranged so that the short arm is on the left side. The locations of the markers on each chromosome are shown at the bottom. Thin lines indicate significant levels for LOD score $=3.0$. Ticks on the $\mathrm{x}$-axis represent $20 \mathrm{cM}$. 


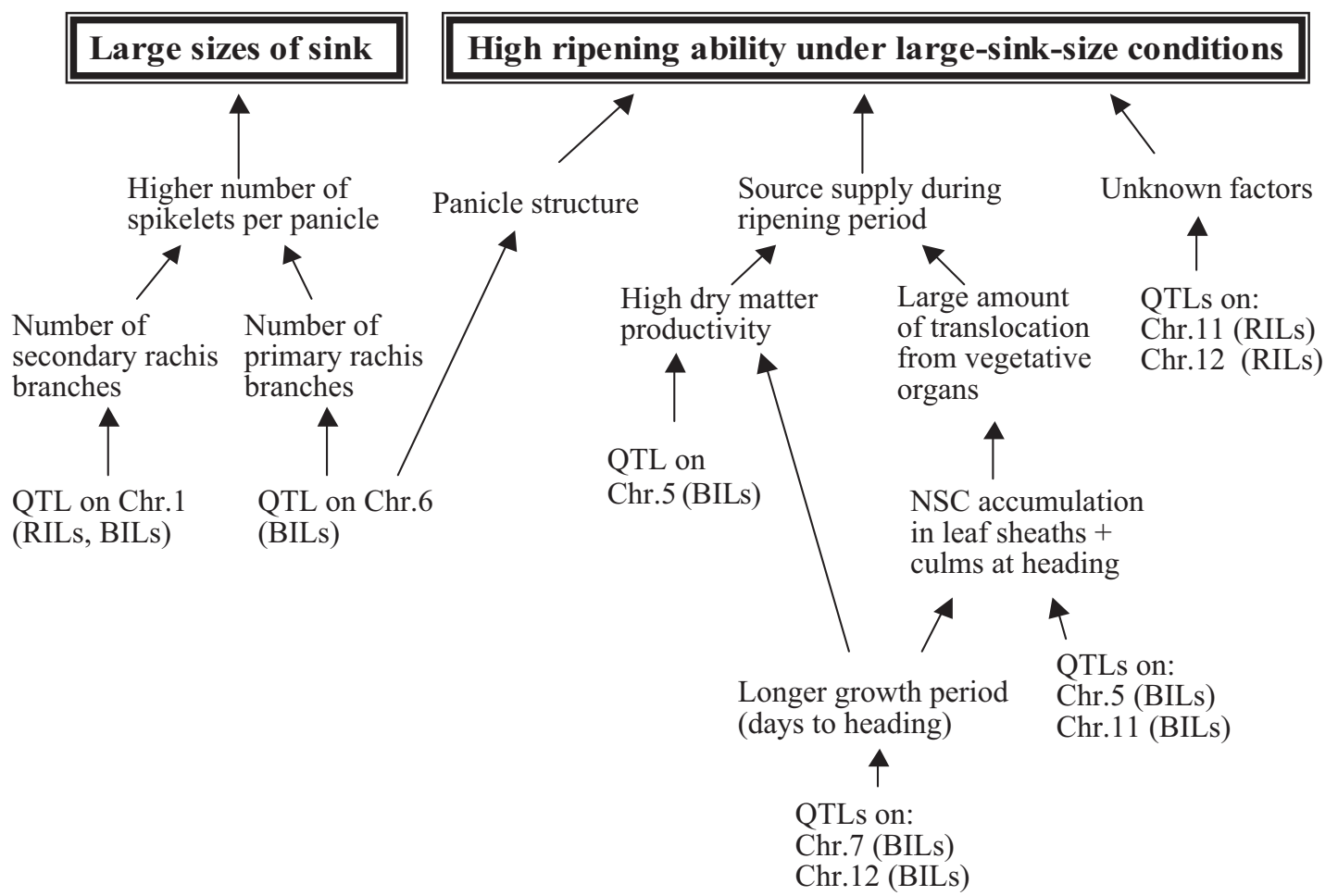

Fig. 7. Relations of the QTLs found in this study with the high-yielding of semi-dwarf indica rice

some-segment substitution lines developed from the BILs used in this study.

Interestingly, the japonica alleles of several regions resulted in increased dry matter accumulation (Figs. 2, 3, $4 \& 5)$. In addition, no changes in the harvest index between semi-dwarf indica and japonica varieties were obtained in our field experiments, as shown in Table 2. These facts might be important keys to the increased yields of the semi-dwarf indica, and it is worth investigating the functions and the effectiveness of these loci in relation to high yields in rice.

\section{References}

1. Chaudhry, F. M. \& Nagato, K. (1970) Role of vascular bundles in ripening of rice kernel in relation to the locations on panicle. Nihon sakumotsu gakkai kiji (Proc. Crop Sci. Soc. Jpn.), 39, 301-309.

2. FAO (2004) FAOSTAT, FAO statistical databases. Available on line at http://faostat.fao.org/.

3. Fukuta, Y. (1995) RFLP mapping of a shattering-resistance gene in the mutant line, SR-1 induced from an indica rice variety, Nan-jing 11. Breed. Sci., 45, 15-19.

4. Fukuta, Y. et al. (1997) Development of Milyang23/Akihikari recombinant inbred lines in rice (Oryza sativa L.). In Proc. 8th SABRAO general congress and the annual meeting of the Korean Breed. Soc., 201-202.

5. Hirayama, T. et al. (1999) Development of $\mathrm{BC}_{2} \mathrm{~F}_{4}$ lines derived from a cross between Sasanishiki and Habataki in rice and construction of RFLP linkage map. Ikushugaku kenkyu (Breed. Res.), 49 (Suppl. 2), 148 [In Japanese].

6. Kabaki, N. (1999) Yield performances and eco-physiological characteristics of newly produced high-yielding rice varieties. In Report of the research project "Development and utilization of improved rice cultivars with diversified and specific grain characteristics", ed. Agriculture, Forestry and Fisheries Research Council Secretariat, MAFF, Tokyo, Japan, 123-136 [In Japanese].

7. Kaneda, C. (1986) Rice breeding for extremely higher yielding ability by Japonica-Indica hybridization. JARQ, 19, 235-240.

8. Komatsu, Y. et al. (1984) Varietal characters of highyielding foreign rice. Shikoku nougyo shikenjou houkoku (Bull. Shikoku Natl. Agric. Exp. Stn.), 43, 1-37 [In Japanese with English summary].

9. Maeda, H. et al. (1997) High density molecular map of semidwarfing gene, $s d-1$, in rice (Oryza sativa L.). Breed. Sci., 47, 317-320.

10. Matsushima, S. (1957) Analysis of developmental factors determining yield and yield prediction in lowland rice. Nougyo gijutsu kenkyujo houkoku (Bull. Natl. Inst. Agric. Sci.)(Ser. A), 5, 1-271 [In Japanese with English summary].

11. Miah, M. N. H. et al. (1996) Characteristics of dry matter production and partitioning of dry matter to panicles in high yielding semidwarf Indica and Japonica-Indica hybrid rice varieties. Nihon sakumotsu gakkai kiji (Jpn. J. Crop Sci.), 65, 672-685. 
12. Nagata, K. et al. (1994) Effects of shading treatments on the growth of high-yielding rice varieties, 1) Changes in dry-matter production process. Nihon sakumotsu gakkai kiji (Jpn. J. Crop Sci.), 63 (Extra issue 2), 91-92 [In Japanese].

13. Nagata, K. et al. (1997) Characteristics of growth and yield of Japanese high-yielding rice varieties cultivated in the Shikoku area. Shikoku nougyo shikenjou houkoku (Bull. Shikoku Natl. Agric. Exp. Stn.), 61, 107-117 [In Japanese with English summary].

14. Nagata, K. et al. (2002a) Quantitative trait loci for sink size and ripening traits in rice (Oryza sativa L.). Breed. Sci., 52, 259-273.

15. Nagata, K., Shimizu, H. \& Terao, T. (2002b) Quantitative trait loci for nonstructural carbohydrate accumulation in leaf sheaths and culms of rice (Oryza sativa L.) and their effects on grain filling. Breed. Sci., 52, 275-283.

16. Sasaki, A. et al. (2002) A mutant gibberellin-synthesis gene in rice. Nature, 416, 701-702.
17. Sumi, A. et al. (1996) Analysis of plant characteristics determining ear weight increase during the ripening period in rice (Oryza sativa L.), II The role of the reserved carbohydrate at heading stage upon the receptive efficiency of assimilation products in spikelets. Nihon sakumotsu gakkai kiji (Jpn. J. Crop Sci.), 65, 214-221 [In Japanese with English summary].

18. Tsukaguchi, T., Horie, T. \& Ohnishi, M. (1996) Filling percentage of rice spikelets as affected by availability of non-structural carbohydrates at the initial phase of grain filling. Nihon sakumotsu gakkai kiji (Jpn. J. Crop Sci.), 65, 445-452 [In Japanese with English summary].

19. Wada, G. (1969) The effects of nitrogenous nutrition on the yield-determining process of rice plant. Nougyo gijutsu kenkyujo houkoku (Bull. Natl. Inst. Agric. Sci.)(Ser. A), 16, 27-167 [In Japanese with English summary].

20. Yagi, T. et al. (2001) QTL mapping of spikelet number in rice (Oryza sativa L.). Breed. Sci., 51, 53-56. 